\title{
VIRTUAL MUSEUMS AS A MEANS FOR PROMOTION AND ENHANCEMENT OF CULTURAL HERITAGE
}

\author{
D. Aiello ${ }^{1}$, S. Fai ${ }^{2}$, C. Santagati ${ }^{1 *}$ \\ ${ }^{1}$ Department of Civil Engineering and Architecture, University of Catania, Italy - cettina.santagati@dau.unict.it, \\ damiano.aiello@gmail.com \\ ${ }^{2}$ Carleton Immersive Media Studio (CIMS), Carleton University, Ottawa, Canada - sfai@cims.carleton.ca
}

\author{
Commission II, WG II/8
}

KEY WORDS: Virtual Reality, Virtual Museums, Cultural Heritage, Digital assets, Playful learning, 3D modelling

\begin{abstract}
:
The use of virtual reality and ICT in the museum context provides a new key to understand and promote Cultural Heritage: thanks to these technologies the user has the opportunity to experience without the need to come into contact with the real objects. For the museum institutions VR and ICT are a valuable tool that allows them to perform different cultural tasks, addressing the public in a much more effective way than has previously been possible. Especially through VR, it is possible to reconstruct the original context of the artworks through the interconnection of contents: the virtual visitor, while viewing the artwork, can consult useful contents for the learning process. Another revolutionary element introduced by the new technologies is linked to the possibility of creating virtual exhibitions through which it is possible to exhibit works that are not accessible or not visible. These reflections and these theoretical principles were the basis for the development of the project proposal presented in these pages, that was born as a collaboration between the $\mathrm{R}^{3} \mathrm{D}$ Lab of the Museo della Rappresentazione of University of Catania and the CIMS Lab of Carleton University, Ottawa. It consists in the creation of a virtual museum, the Timeless Museum, in order to create an educational experience, able to make the users reflect on topics such as the value of history, the sense of beauty, the relationship with our past and our future, the protection and transmission to future generations of the artistic heritage we have.
\end{abstract}

\section{INTRODUCTION}

We are living in the age of Industry 4.0. It's impact in the field of Cultural Heritage is under our eyes and is still to be assessed. The potentialities of Virtual Reality (VR) and Mixed Reality (MR) technologies are undoubtedly opening new perspectives for experiencing cultural heritage in terms of visualization, representation, interaction, exploitation, enhancement and dissemination. With particular reference to museums and cultural and historical sites, the benefits to the Creative Industry sector can be envisaged in the possibility of addressing new audiences, introducing new means of interaction, creating new engaging stories to tell, changing and innovating our perception and understanding of cultural assets.

This research is part of a broader reflection on the potentialities of immersive technologies (VR/MR), with a focus on Virtual Museums (VM) and the use VR for as a means for creating educational environment, thus augmenting or accessing a better learning, communication and entertainment of cultural spaces.

The research group has explored the most modern theoretical definitions related to the concept (still fluid and being developed) of VM and has tested several workflows linked to the technical issues related to the combined use of modeling software, game engines and online platforms for sharing 3D content. In this regard, a complete workflow has been developed that goes from $3 \mathrm{D}$ modeling to the creation of a complex VR simulation, characterized by a captivating storytelling capable of transmitting a profound educational content.

The experimentation has led to the creation of a prototype of a VM, named Timeless Museum, which is inspired by the paradigm of playful learning and explores VR's ability to convey an emotional knowledge of the art masterpieces of all times, bringing together in the same virtual place works exhibited in physically distant museums.

Another relevant topic addressed in this study is the unlimited potential in terms of creativity that the reuse of digital assets available on the web (Europeana, Sketchfab, etc.) could have in the creation of products or Apps for the promotion and enhancement of cultural heritage, as well as the possibility of rethinking the physical space of the museum involving the virtual visitor in an all-encompassing way and simultaneously breaking the traditional conception of exhibition space.

Overall, this virtual museum prototype tries to make a significant contribution to the current debate on the role of Virtual Museums, the importance of new learning methods and the formation of increasingly specialized and transversal skills in the creative industry.

The designed and developed prototype is installed at the Museo della Rappresentazione, a University museum of the Department of Civil Engineering and Architecture of University of Catania. For assessing the effectiveness of the museum, an evaluation form has been carried out and is submitted to the visitors.

The paper is structured as follows: section 2 will deepen the definition of VM and an overview of significant project; section 3 to the design of the VM; section 4 to the workflow used to create the virtual simulation; section 5 to the user experience evaluation; section 6 to conclusion and future research development.

\footnotetext{
* Corresponding author
} 


\section{RELATED WORKS}

The spread, in recent years, of immersive technologies in the field of cultural heritage has led to a revolution in the way we think the museum and museum exhibitions. Indeed, ICT provides new and unconventional opportunities for learning, communication and entertainment in the sector of Cultural Heritage. As a consequence, today, ICOM is reshaping the definition of museum, embedding concepts related to the key role that technology can play.

The increasingly advances in terms of technology, experimentations and applications lead to a still fluid and under development definition of VM (Perry et al, 2017; Povroznik, 2018). A first attempt to define it in a systematic manner can be found in the outputs of the EU funded project V-MUST (Pescarin, 2014; Hazan et al., 2014).

The ViMM project ( https://www.vi-mm.eu/) has recently published on Wikipedia (https://en.wikipedia.org/wiki/ Virtual_museum) a revised definition of what a VM is: a digital entity that draws on the characteristics of a museum, in order to complement, enhance, or augment the museum through personalization, interactivity, user experience and richness of content. Both the 'physical' museum $(\mathrm{PhM})$ and the VM share a common commitment to the institutional validation of content and quality of experience through curatorial process, inherent in the ICOM definition.

In literature it is possible to find several research projects and applications that draw on the endless declinations of VM, each of them based on one or more aspects.

Many projects are focused on the digitalization of a cultural site (Remondino et al, 2018) and its virtual exploration in an immersive way (Dhanda et al 2019; Choromariski et al 2019, Paladini et al, 2019), making it possible, in some cases, the accessibility to people with disabilities (Pèrez et al, 2019). Other experimentations are aimed at the digital preservation of heritage sites at risk; in this way, the VM constitutes an educational product and an alternative way for visiting the site, relieving human pressure (Aiello et al, 2019; Tschirschwitz et al, 2019). Another interesting development is the possibility of allow the combination of exhibits physically distant from each other, or the exhibition of objects that have no physical presence and are born digital (Kazanis et al, 2017). Many projects address the digital reconstruction and recontextualization of artefacts that no longer exist (Anastasovitis. E., Roumeliotis, 2018; Fanini et al, 2019; Agnello et al, 2019) also allowing an understanding of the timeline (Kersten et al, 2017).

All the cited experimentations of VM are valuable examples of how the concept idea is devoted to augment the educational engagement of the visitor, proposing stimulating forms of storytelling in an immersive and interactive environment (Pietroni et al, 2018; Latos et al, 2018).

Furthermore, often these VM prototypes are oriented towards a Serious Game approach with a balanced presence of three components: educational, simulative and playful.

Our approach is focused on the exploration of the concept of $\mathrm{VM}$, which takes the form of a timeless place where items coming from different collections can be put together, in order to tell the story of the Mediterranean culture, through an out of scheme interpretation of places and exhibition rooms.

\section{DESIGNING A VM: THE TIMELESS MUSEUM}

\subsection{Design concept}

The preliminary concept design of the VM has taken into account the idea of creating a virtual educational experience, able to make the users reflect on topics such as the value of history, the sense of beauty, the relationship with our past and our future, the protection and transmission to future generations of the artistic heritage we have.

Considering the infinite creative possibilities that the designer encounters projecting a virtual dimension, several aspects have been addressed:

\section{Design of the virtual space \\ Selection of the items to exhibit \\ Exposition criteria \\ Design of the educational experience}

Thus, it has been given an adequate space to the expressive freedom allowed by Virtual Reality (Ferriozzi, Valenti, 2016), giving life to a place strongly characterized by interactive elements and in which the visit is enriched by experiences that it would not be possible to live in the real world (e.g. seeing some of the greatest masterpieces of human history gathered in the same place, observing them at unimaginable distances in the real world, receiving information from interactive panels that appear automatically when the visitor approaches them, etc.).

The Timeless Museum is envisioned as a virtual space where a selection of some of the most iconic artworks of the Mediterranean history are shown in such a sequence and a way as to engage visitors in a captivating educational experience, full of sensorial stimuli. The final goal of this virtual exhibition is to deliver an ethical message to the visitor within a dystopian dimension that shows an apocalyptic future where the most emblematic symbols of cultural heritage have been destroyed.

\subsection{VM exhibition}

As mentioned, the Timeless Museum illustrates some of the most emblematic periods in human history, starting from the Egyptian civilization, passing through the Greek and Roman art and finally arriving at a possible future. This is why four sections (an Egyptian one, a Greek one, a Roman one, a gallery) and a dystopic dimension have been designed.

The visit starts from an entrance hall, lit by a large window and open onto a great central courtyard garden (Figure 1).

From here the visitor is directed towards the corridor where there are the four sections of the museum, all arranged along an evocative circular path where the beginning and end coincide. These sections are designed as "other worlds" that somehow revive the spirit of the historical epoch exposed. For this purpose, the entry of each of these sections is configured almost like a temporal portal that announces the passage from the atrium - located outside of space and time - to the various environments. 


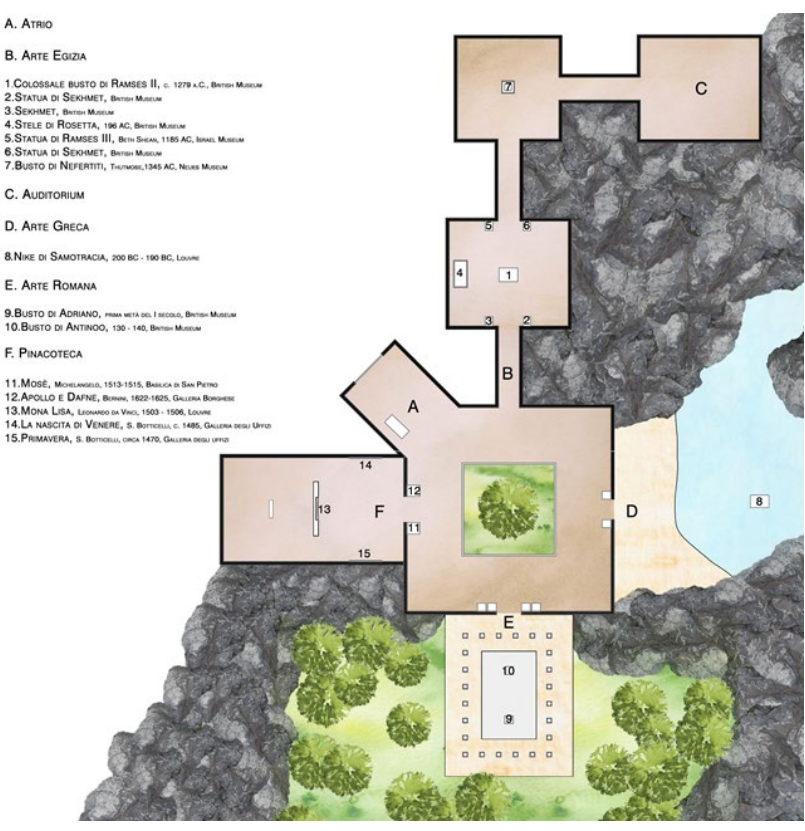

Figure 1. Plan of the Timeless Museum

The entrance of the Egyptian section (Fig. 2) is marked by a large stone trilith. The enigmatic solemnity of this great opening begins to unveil itself when the view goes along the narrow space after the portal: at the bottom, immersed in an atmosphere of silence and penumbra that aims to evoke the sacredness of Egyptian temples and sacred funeral rooms, there are quadrangular environments, connected by narrow and long straight corridors. The rooms in this section host some of the most emblematic works of Egyptian art (as the great statue of Ramses II, the bust of Nefertiti and the Rosetta Stone).

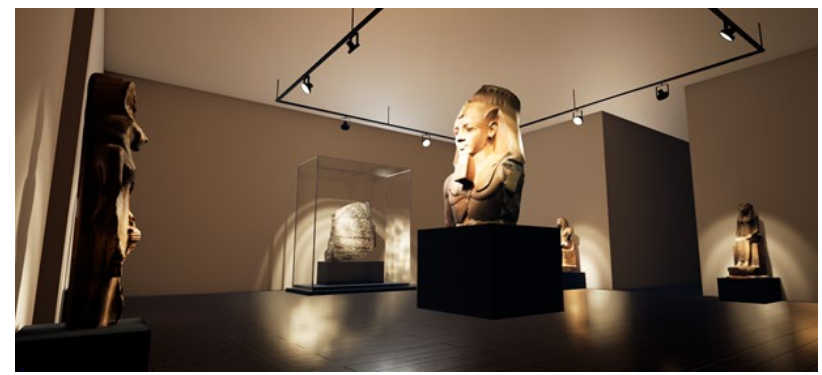

Figure 2. Egyptian section

The Greek section (Fig. 3) is indicated by two Ionic columns surmounted by an architrave, which introduce into a dimension that is halfway between history and myth: the bay that extends beyond the portal was designed to recall the waters from which the goddess Aphrodite was created, as also the Anatolian coast where, in $190 \mathrm{BC}$, the naval battle of the Eurymedon took place, at the end of which the city of Samothrace - to commemorate its victory against the Syrian king Antiochus III - commissioned the Winged Victory of Samothrace, which stands here on the water, in an attempt to recall the historical event.

The vestiges of a triumphal arch introduce to the Roman section (Fig. 4). Here there is a garden, in the center of which the remains of a Roman architecture have been modeled to recall a domus peristyle. At the center of the colonnade were placed two marble busts depicting the emperor Hadrian and Antinous, a young Greek native of Bithynia who, according to the chronicles, was the emperor's lover and was deified by the emperor after his death. The sense of immersion is emphasized by the bucolic setting that surrounds the ruins. The scene is also animated by the presence of the wind that moves the leaves of the trees (creating suggestive plays of shadow) and the sounds of nature.

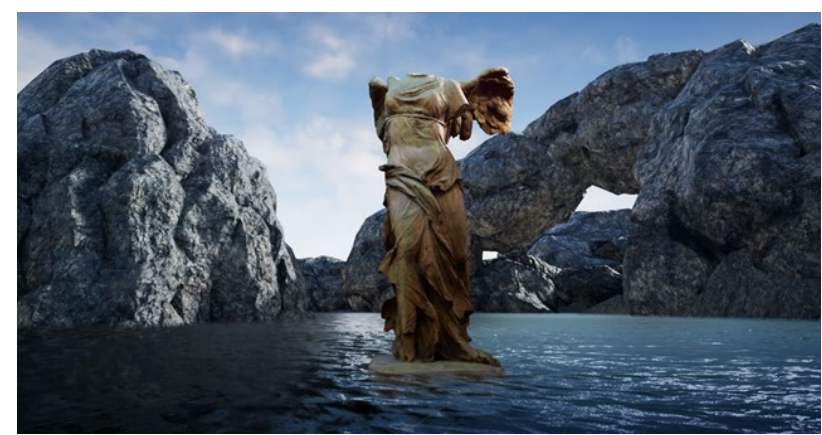

Figure 3. Greek section

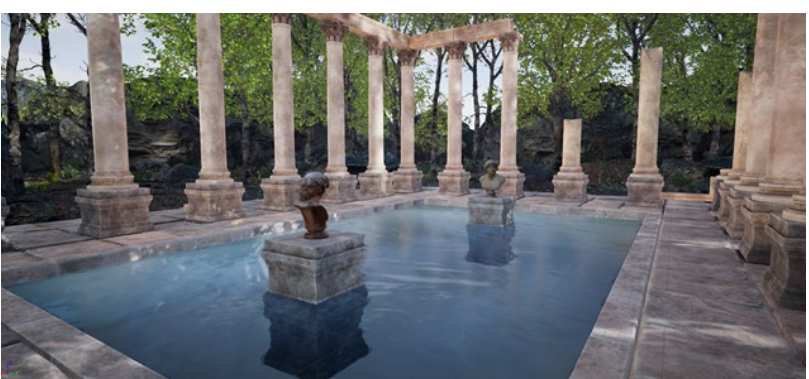

Fig. 4. Roman section

The gallery (Fig. 5) is marked by two sculptures, the Moses by Michelangelo and the Rape of Proserpina by Bernini, representing respectively the late Renaissance and the Baroque art. This section holds some of the most iconic artworks of the modern age. The great hall hosts the works of Sandro Botticelli, Primavera and The Birth of Venus, and the most famous work of Leonardo da Vinci, the Mona Lisa. Gioconda's enigmatic charm has been chosen as an indicator to signal the presence of a mysterious element (with the aim of giving the virtual simulation a playful component in the ways of serious games dimension) hidden beyond the wall, i.e. a dimensional portal that allows to project the user in the last dimension, the dystopian future.

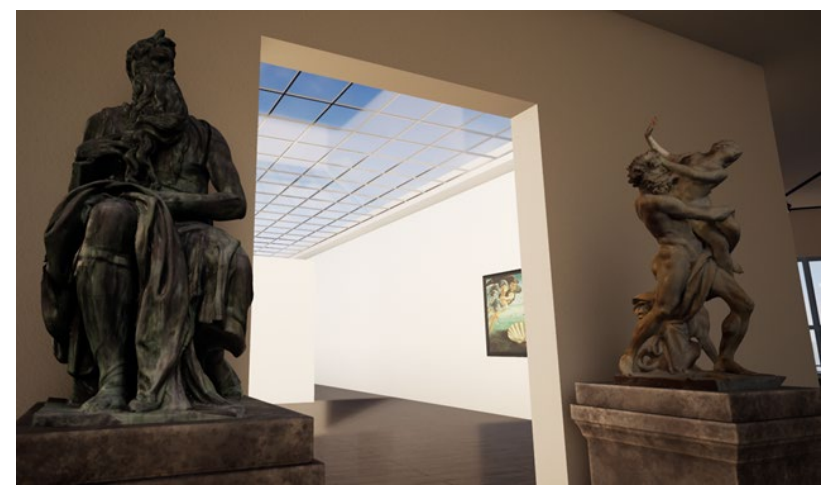

Figure 5. Gallery

Beyond the arch, in fact, the visitor is projected into a new dimension that is characterized by different traits from those already seen: the appearance of the ruins and the presence of three large holograms characterize this place as a sample of a possible future. The gloomy atmosphere and the constant background noise contribute to increase the sense of alienation and restlessness. The three holograms represent three of the best-known architectures of our present (the Eiffel Tower, the 
Statue of Liberty and the $\mathrm{CN}$ Tower) which no longer exist in this alternative future because they have been destroyed due to violence, divisions, self-destruction's instinct, fanaticism, which prevailed over reason, ability to create, love for beauty and for all that makes us authentically human (Fig. 6).

At this point the visit ends with the ideal delivery of the final message to the visitor, in front of which a video (extracted from some original journalistic reports) portrays ancient monuments and works of art destroyed by ISIS militias. The visitor is thus called to reflect and wonder whether the dystopian future in which he is in would not be his present.

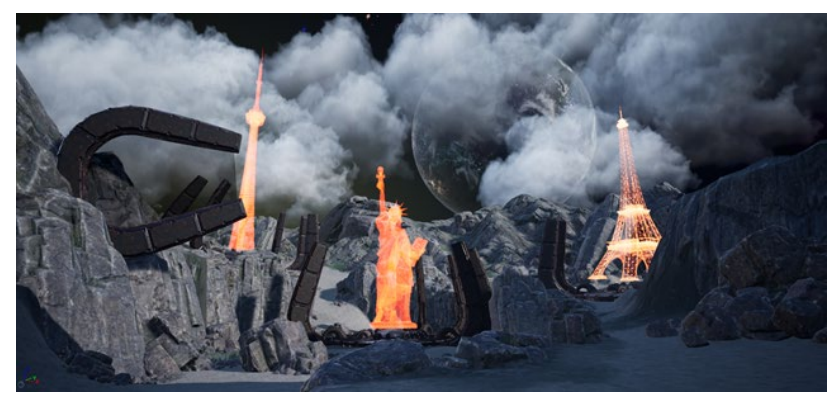

Figure 6. Dystopian dimension

\subsection{Immersion and interactivity in the VR simulation}

To make the virtual experience effective and intense, great attention has been paid to how the visitor interacts with the virtual environment. This interaction takes place in different ways and at different levels; the first type of contact with the virtual dimension (which is also the first and the most immediate) is the visual one: it is linked to the perception of the immersive virtual dimension, which entirely envelops and surrounds the visitor. The second level of interaction (less immediate but more intense) is the manipulation of objects: from the beginning of the visit, in fact, the visitor can actively relate with various objects arranged in the museum rooms, starting from the pamphlet placed at the entrance hall (where the building plan and the legend of the exhibition are shown), or the artworks (approaching them information panels appear to give more historical notions about the masterpiece). The last mode of interaction involves the auditory sphere. The presence (as well as the absence) of sounds everywhere has its raison d'être and helps to make the virtual experience more realistic, thus making the user the active protagonist of the museum. The main sound component is represented by a narrator voice, which guides the visitor along the path, indicating the right direction to follow, explaining how to get in touch with interactive objects, telling stories and anecdotes, highlighting the characteristics and symbols hidden behind the exhibited artworks, finally arriving to unveil and ideally give the visitor (arrived at the end of his visit) the deepest educational message of the museum. Other sounds (or other non-random sound absences) enrich the auditory component (the hieratic silence in the rooms dedicated to ancient Egypt, the lapping of the waves in the Greek world, the sounds of nature in the Roman ruins, the dark background noise in the dystopian dimension).

\section{ASSESSING A WORKLFOW FOR THE CREATION OF THE VR SIMULATION}

\subsection{VM modelling and reuse of digital assets}

The two main components of the museum, namely the architectural envelope and the exhibited objects, have been realized through different procedures.
As for the most important architectural elements (walls, floors, ceilings, windows, openings, corridors, etc.), they were modeled in the form of NURBS objects within the 3D modeling software Rhinoceros.

Instead, the artworks hosted in the virtual museum were obtained in two ways: a part of them was downloaded from the web, through Sketchfab, a platform that allows you to view (even in virtual reality), upload, share and download 3D models as static or animated mesh and as point clouds, without the user having to install any software. As regards the paintings in the gallery, they have been reconstructed from images applied as textures on solids modeled in Rhino.

From the above, it appears that the virtual museum is configured as a tool able to guarantee the reuse of digital assets. Indeed, the creativity and versatility of the virtual museum allow to repurpose and reimagine the multimedia contents present on the web in new ways by organizing digital assets in a flexible and an orderly manner, making them easy to find and easy to distribute.

\subsection{Workflow for optimizing digital assets}

The models downloaded from the web (such as those provided by the British Museum) are meshes obtained through photogrammetry. These type of meshes are difficult to manage within the VR software: in fact, the large number of triangular polygons that make up these meshes (together with the many interactions that are usually created in VR) tends to considerably reduce the number of frames per second (fps) that we observe in the headset during the virtual experience. VR software works actually as a real-time rendering engine, and consequently it has difficulty in quickly rendering very complex objects or interactive elements, so when our eyes focus on them, the scene inside the viewer can appear slow and not very fluid. To avoid this, it is necessary to simplify the meshes as much as possible, so that the frames per second in the display are not less than 60 (the optimal value is between 60 and $90 \mathrm{fps}$ ).

For this reason, we applied a workflow for their optimization within ZBrush, a digital sculpting tool that combines 3D/2.5D modeling, texturing and painting. The optimization is essentially based on a procedure for redefining the topology of the model, an operation that is called retopology (Merlo et al, 2012; Palestini, Basso, 2019). The topology of a threedimensional object coincides with its internal geometry and is determined by the number and shape of the faces that constitute it. Consequently, changing the topology of a mesh essentially means reducing the number of its faces (so as to have only those strictly necessary) and modifying their triangular geometry. For this reason, we prefer to generate new meshes, which trace as much as possible the starting triangular surfaces but which are made up of an optimized number of quadrangular faces, easier to manipulate in sculpting programs or to render in real time in the VR game engine.

Once the new quadrangular meshes are obtained, the UV maps are generated, through a procedure that takes the name of unwrapping. Unwrapping is the process of translating a 3D mesh into a $2 \mathrm{D}$ representation. This requires unfolding the mesh in correspondence of one or more seams, that can be traced manually or through an automatic process. Thanks to a Zbrush plug-in (UV Master), it is possible to identify the seam along which to unwrap the mesh. UV Master packs the created UVs to optimize the UV space. It scales, rotates and moves them to use the maximum space, but it always maintains the ratio between the UVs and the polygons.

Anyway, user can manually change the location and the size of the UVs to best fit the space of the map. Through this process it 
is possible to create an optimized UV map thanks to which the texture maps (containing the color information) and the normal maps (containing the roughness information) can be obtained. The textures of the triangular models (with their high level of detail), are projected onto the new quadrangular surfaces. In this way, although the meshes have been significantly decimated during the optimization phase, (thus losing a lot of information on their morphology) their apparent level of detail has not been lost, since it has been extrapolated from the texture of the starting triangular models (Fig. 7).
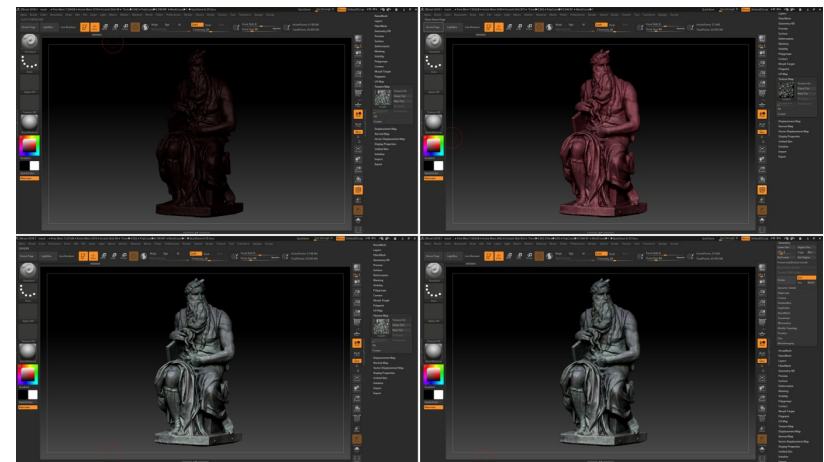

Figure 7. On the left, triangular model made up of $6 \mathrm{ML}$ of triangular polygons. On the right, a retopologized model consisting of 57646 quadrangular polygons

\subsection{VR Game Engine}

Once the museum's architecture was completed and all the meshes were optimized, the entire model was exported in fbx. The research group then tried to understand which was the most appropriate game engine in which to import the model, with the aim of creating the interactive virtual simulation.

In the present experimentation, the researchers will refer in particular to Unreal Engine (Fig. 8). The choice of Unreal Engine for the creation of the Timeless Museum is due to the software's versatility, the availability of a free version, the reliability of the results obtained and the possibility of drawing on vast libraries of materials and a substantial literature produced during the years in which the use of the software has been consolidated. One of the strengths of this tool is the visual scripting system Blueprint, constantly updated and so powerful that it can replace programming languages like $\mathrm{C}++$ in very complex operations. In addition, Blueprint-specific markup available in Unreal Engine's C++ implementation enables programmers to create baseline systems that can be extended by designers.

Blueprints are visually scripted additions to the game. By connecting Nodes, Events, Functions and Variables with Wires, it is possible to create complex gameplay elements.

The most commonly used Blueprint types are "Level Blueprints" and "Blueprint Classes".

The first type is specific to the level of the Unreal Editor in which Level Blueprints were developed, so they are especially suitable for creating unique actions that should not be repeated: start a movie when a certain object is touched or open a particular door after completing an action. It can reference and manipulate Actors within the level, control cinematics (using Matinee Actors), and manage things like level streaming, checkpoints, and other level-related systems. Level Blueprints can also interact with Blueprint Classes placed in the level, such as reading/setting any variables or triggering custom events they might contain.

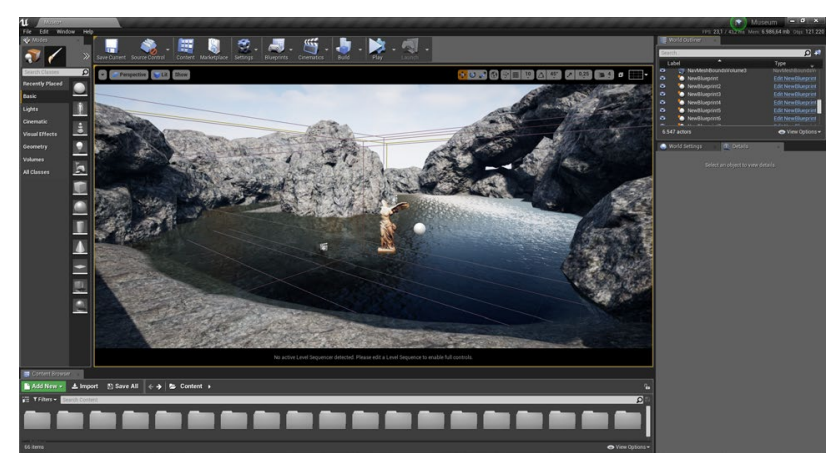

Figure 8. View of the virtual model within Unreal Engine

On the other hand, Blueprint Classes are the best way to get a reusable behavior in the project: because of the self-contained nature of Blueprints, they can be constructed in such a way that they can be dropped into a level and they will simply work, with minimal setup required. This also means that editing a Blueprint that is in use throughout a project will update every instance of it. They are ideal for creating interactive assets such as doors, switches, playable sounds etc.

In the Timeless Museum, Blueprint Classes were used to create the majority of the interactions (such as picking the pamphlet up at the entrance, displaying information panels next to the artworks, allowing teleportation from the art gallery to the dystopian dimension through the dimensional portal, observing the video contents, etc.) (Fig. 9).

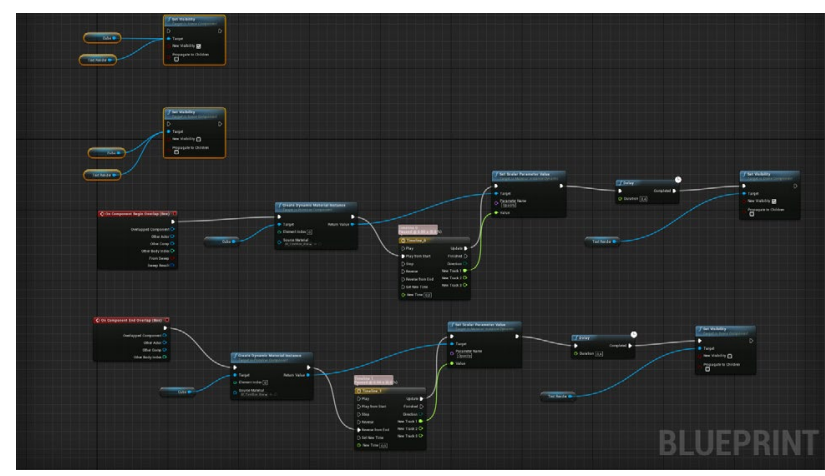

Figure 9. Blueprint Class of the info panel

As regards some particular interactions, such as the narrative voice, they were made using Level Blueprints (Fig. 10).

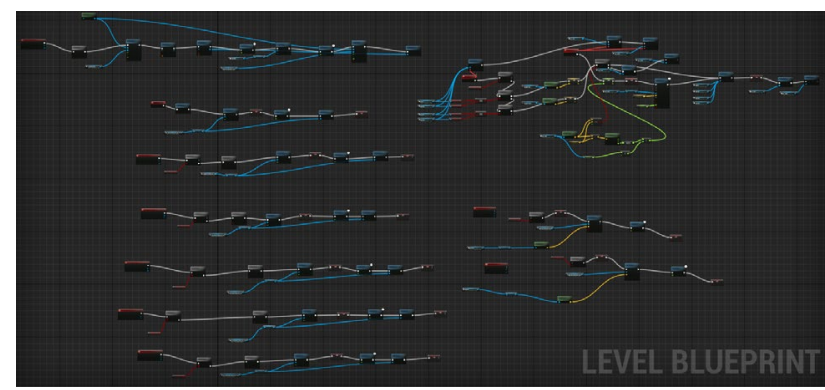

Figure 10. Level Blueprint of the narrative voice

These operations have been facilitated, as mentioned, by the possibility offered by Unreal Engine to build interactions through connections between nodes, without directly using the $\mathrm{C}++$ language codes. 


\subsection{Adopted VR Technologies - Head-Mounted Display}

In order to experience the VM created in this experimentation, it was decided to use an Oculus Rift device (developed by Oculus VR), falling into the category of Discrete HMD (HeadMounted Display). An HMD consists of a stereoscopic display that returns images to the user as they are perceived by the human eye: two images of the same scene with two slightly different points of view are projected into each eye to create the parallax effect, the phenomenon which makes the brain perceive depth, based on the apparent position of objects. Oculus Rift is a fully immersive display, as it completely blocks the view of the user and prevent any visual contact with the surrounding world.

In detail, the Oculus Rift CV1 (Consumer Version-1) is a lightweight stereoscopic display with a wide field of view (FOV) of $110^{\circ}$, mounted on two low latency AMOLED flat screens with $1080 \times 1200$ resolution per eye. Of particular importance in this display is the use of highly specialized and free-form hybrid Fresnel lenses.

The position and orientation of the device (positional and rotational tracking) are traced using the combination of an internal Inertial Measuring Unit (an electric sensor located inside the HMD consisting of a gyroscope, an accelerometer and a magnetometer) and a series of infrared LEDs (incorporated in the display and in the support strap) which are detected by a separate IR camera. The system is designed for the use in an upright or sitting position and has an effective operating area measuring $5 \times 11$ feet. The position and orientation of the controllers are monitored using the Oculus Constellation tracking technology, consisting of an infrared camera that identifies the position of the LEDs arranged around each device. The user can navigate in the virtual environment using two controllers (Oculus Touch). The inputs of the controllers, such as those that detect the position of the user's fingers, are sent via a wireless transmitter to a receiver in the HMD (Aukstakalnis, 2016).

\section{USER EXPERIENCE EVALUATION}

In order to evaluate the effectiveness of the work, understand the way users experience the virtual simulation and find ideas to improve it, an evaluation questionnaire was prepared and submitted to a sample of 50 users ( 25 women and 25 men, aged between 13 and 65, with different educational levels and with different technological skills levels took part in the first evaluation).

The questionnaire consists of a first part to identify the sample (gender, age, origin, educational qualification, employment) and its skills (skills in the use of technologies, technological tools used, previous experiences in the field of virtual reality).

The second part of the test specifically concerns the experience lived in the Timeless Museum and proposes a series of questions aimed at understanding the level of satisfaction (duration of the visit, ease in finding information, visited sections, most pleasant sections, museum's organization, any suggestions).

In general, the sample expressed a positive opinion about the visit as a whole. Specifically, $82 \%$ of the total showed the highest level of satisfaction and the desire to repeat the experience in the future (Fig. 11).



Figure 11. Level of satisfaction of the virtual experience

The test also revealed the museum's usefulness as regards its information content: the exhibition's organization, the information panels associated with each artwork, the narrator voice that guides users and the multimedia contents have provided $86 \%$ of the sample with new knowledge (Fig. 12).

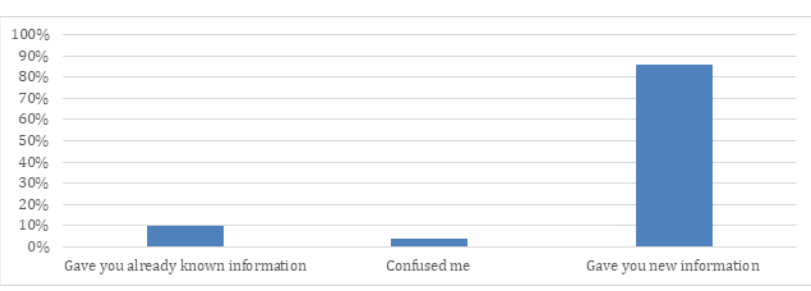

Figure 12. General satisfaction of the information gathered during the virtual visit

The evaluation of the exhibition rooms has provided interesting causes for reflection. In particular, the section that hosts Egyptian art has turned out to be the one that had the greatest success, which could be an indication of the fact that in the considered socio-cultural context there is still a strong attachment to a traditional idea of museum, consisting of exhibition rooms designed as containers for works of art.

Despite this result, the other sections of the museum also aroused significant interest (Fig. 13). In this regard, the dystopian dimension, with its strong ethical content, was particularly appreciated. This allowed to understand that the idea to further develop the VM in the direction of the Serious Game goes in the right direction.

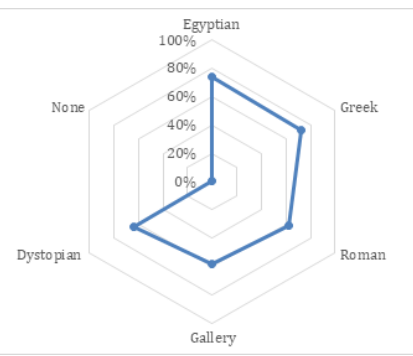

Figure 13. Evaluation graphic: which section did you prefer?

Certain feedbacks showed their relationship with the age and the educational level of the sample. For example, the level of awareness related to the use of new technologies applied to the cultural promotion is directly proportional to the level of education: $89 \%$ of graduates think that new technologies can and should be used to promote culture against $82 \%$ of high school graduates and $60 \%$ of those with a primary or middle school degree.

As regards the appreciation of the virtual experience, it was found that the answer is more correlated with the sample's age: $91 \%$ of people under 18 expressed the maximum index of approval, against $81 \%$ of the sample between 18 and $30,71 \%$ of 
the sample between 31 and 45 and $82 \%$ of the sample between 46 and 65 . If the high interest from younger people may seem obvious (considering their familiarity with technological tools), it positively strikes the high percentage of appreciation from the highest aged groups, a probable sign of the fact that the use of ITCs is taking root more and more even among old aged users.

The questionnaire also revealed interesting suggestions regarding the improvement of the museum's storytelling, the exhibition path and the interactive components. The proposals made include:

- $\quad$ adding indicators to make the visit clearer;

- adding other interactive elements that can be handled by the user;

- adding more information in the interactive panels.

From other comments, it seems that a part of the sample expected a more present playful component, to the detriment of the information element.

These data are very valuable because they provided researchers with new foods for thoughts and new ideas to improve the visiting experience of the Timeless Museum, increasing its interactive component and its serious game character.

The second evaluation was attended by a sample of 20 users (10 men and 10 women) aged between 18 and over 65 , with a prevalence of the medium-high aged group. In this case, the level of education was more homogeneous, with a prevalence of higher level of education.

Here too, the sample expressed itself positively on the VR experience: $85 \%$ of the total showed the highest level of satisfaction (Fig. 14).

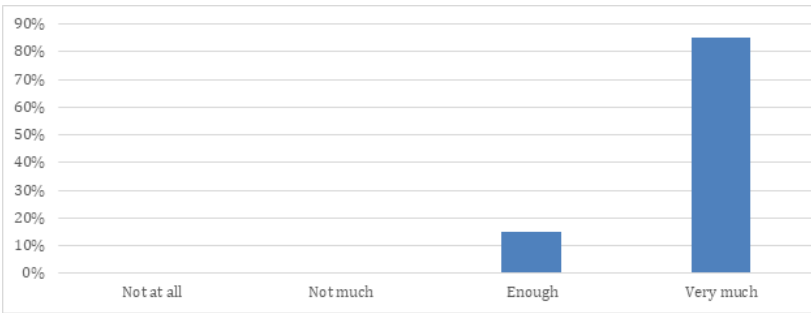

Figure 14. Evaluation graphic: are you satisfied of the virtual experience?

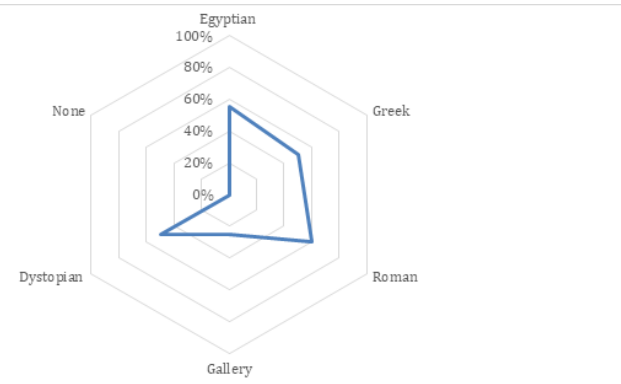

Figure 15. Evaluation graphic: which section did you prefer?

The whole sample agreed that the experience provided them with new knowledge, compared to $86 \%$ in the previous case. As regards the level of appreciation shown for the exhibition rooms, it was more uniform than that emerged from the first evaluation, although in this case there was a slight prevalence of the Roman section compared to the others (Fig. 15). This could indicate a greater sensitivity towards virtual reality from people who have a higher educational level and, for this reason, show the willingness to abandon the patterns imposed by the traditional museum in order to embrace unconventional exhibition solutions.
Here again, finally, suggestions were made to improve the Timeless Museum. Among these, a teacher's proposal to provide the use of VR experiences in schools has attracted considerable attention. What emerged seems to corroborate the idea that virtual reality finds fertile ground in the field of education; the serious game is configured as a valid tool for teaching, transmitting a message, helping younger people to reflect on topics of high ethical content (e.g. approaching culture and respecting art).

\section{CONCLUSION AND FUTURE RESEARCH}

This work has set itself the ambitious goal of investigating the influence of virtual reality applied in the field of protection, conservation, enhancement and communication of cultural heritage. The VM designed and developed demonstrate the powerful potentialities between the playful component and the educational one in a totally immersive environment.

Through an exhibition and a storytelling that aim to be interesting and exciting, the user, guided through the museum sections (more similar to worlds-others), lives an immersive brand experience which helps him to learn the information contained in the museum and to discover the educational message that it contains. The Timeless Museum is currently under evaluation at the "Museo della Rappresentazione" (University of Catania). A sample of 70 users provided positive feedback, also proposing food for thought and useful suggestions for a possible future development and for a global improvement of the virtual experience. What is clearly evident from this first test seems to support the idea that virtual reality finds fertile ground in the field of education; serious game is configured as a valid tool for teaching, transmitting a message, helping young people to reflect on issues of high ethical content such as the approach towards culture and the love for art.

In conclusion, the research has showed the creative ways in which virtual reality applied to serious games makes cultural education possible and has highlighted VR's ability to bring people closer to contexts that are somehow distant from them. This last reflection has significant implications, especially in reference to the categories of people who, living in alienated and alienating contexts, do not have the possibility to travel and, without a technology such as virtual reality, could not enjoy extraordinary places and live extraordinary experiences.

\section{ACKNOWLEDGEMENTS}

This work has been partially financed by the University of Catania within the project "Piano della Ricerca Dipartimentale 2016-2018" of the Department of Civil Engineering and Architecture.

\section{REFERENCES}

Agnello, F., Avella, F., Agnello, S., 2019. Virtual Reality for historical architecture. In: Int. Arch. Photogramm. Remote Sens. Spatial Inf. Sci., Vol. XLII-2/W9, pp. 9-16, doi.org/10.5194/isprs-archives-XLII-2-W9-9-2019.

Aiello, D., Basso, A., Spena, M. T., D'Agostino, G., Montedoro, U., Galizia, M., Grasso, R., Santagati, C., 2019. The Virtual Batcave: A Project for the Safeguard of a Unesco WHL Fragile Ecosystem. In: Int. Arch. Photogramm. Remote Sens. Spatial Inf. Sci., Vol. XLII-2/W9, pp. 17-24, doi.org/10.5194/isprs-archives-XLII-2-W9-17-2019.

Anastasovitis, E., Roumeliotis, M., 2018. Virtual Museum for the Antikythera Mechanism: Designing an Immersive Cultural 
Exhibition. In: IEEE International Symposium on Mixed and Augmented Reality Adjunct (ISMAR-Adjunct), doi: 10.1109/ISMAR-Adjunct.2018.00092.

Aukstakalnis, S., 2016. Practical Augmented Reality: A Guide to the Technologies, Applications, and Human Factors for AR and VR. Addison-Wesley Professional, Boston.

Choromański, K., Łobodecki, J., Puchała, K., Ostrowski, W., 2019. Development of Virtual Reality application for Cultural Heritage visualization from multi-source 3D data. In: Int. Arch. Photogramm. Remote Sens. Spatial Inf. Sci., Vol. XLII-2/W9, pp. 261-267, doi.org/10.5194/isprs-archives-XLII-2-W9-2612019.

Dhanda, A., Reina Ortiz, M., Weigert, A., Paladini, A., Min, A., Gyi, M., Su, S., Fai, S., Santana Quintero, M., 2019. Recreating cultural heritage environments for VR using photogrammetry. In: Int. Arch. Photogramm. Remote Sens. Spatial Inf. Sci., Vol. XLII-2/W9, pp. 305-310, doi.org/10.5194/isprs-archives-XLII2-W9-305-2019.

Fanini B., Pagano A., Palombini A., 2019. The Virtual Museum of the Upper Calore Valley. In: Luigini, A. (ed) Proceedings of the 1st International and Interdisciplinary Conference on Digital Environments for Education, Arts and Heritage. EARTH 2018. Advances in Intelligent Systems and Computing. Springer, Cham, pp. 726-736.

Feriozzi R., Valenti G.M., 2016. Parametric Procedures to Create Multi-dimensional Virtual Museums. DISEGNARECON. 9 (17), 13.1-13.9.

Hazan, S., Hermon, S., 2014: On defining the Virtual Museum. A VMUST research project. In Cappellini V. (ed) EVA Florence 2014. 30-35

Kazanis, S., Kontogianni, G., Chliverou, R., Georgopoulos, A., 2017. Developing a Virtual Museum for the ancient wine trade un Eastern Mediterranean. In: Int. Arch. Photogramm. Remote Sens. Spatial Inf. Sci., Vol. XLII-2/W5, pp. 399-405, doi.org/10.5194/isprs-archives-XLII-2-W5-399-2017.

Kersten, T. P., Tschirschwitz, F., Deggim, S., 2017. Development of a Virtual Museum Including a 4D presentation of building history in Virtual Reality. In: Int. Arch. Photogramm. Remote Sens. Spatial Inf. Sci., Vol. XLII-2/W3, pp. 361-367, https://doi.org/10.5194/isprs-archives-XLII-2-W3361-2017.

Latos, A., Komianos, V., Oikonomou, K., 2018. Interaction and Information Communication in Virtual Museums. In: IOP Conf. Ser.: Mater. Sci. Eng., 364012038.

Merlo, A., Dalcò, L., Fantini, F., 2012. Game engine for Cultural Heritage: New opportunities in the relation between simplified models and database. In: 18th International Conference on Virtual Systems and Multimedia, doi.org/10.1109/VSMM.2012.6365993.

Paladini, A., Dhanda, A., Reina Ortiz, M., Weigert, A., Nofal, E., Min, A., Gyi, M., Su, S., Van Balen, K., Santana Quintero, M., 2019. Impact of Virtual Reality experience on accessibility of Cultural Heritage. In: Int. Arch. Photogramm. Remote Sens. Spatial Inf. Sci., Vol. XLII-2/W11, pp. 929-936, doi.org/10.5194/isprs-archives-XLII-2-W11-929-2019.
Palestini, C., Basso, A., 2019. 3D detection and reconstruction experiments in river basins. In: Int. Arch. Photogramm. Remote Sens. Spatial Inf. Sci., Vol. XLII-2/W9, pp. 543-550, doi.org/10.5194/isprs-archives-XLII-2-W9-543-2019.

Pérez, E., Merchán, M. J., Salamanca, S., Merchán, P., 2019. Virtual Reality to allow wheelchair users touring complex archaeological sites in a realistic manner. Towards their actual social integration. In: Int. Arch. Photogramm. Remote Sens. Spatial Inf. Sci., Vol. XLII-2/W9, pp. 597-604, doi.org/10.5194/isprs-archives-XLII-2-W9-597-2019.

Perry, S., Roussou, M., Economou, M., Pujol-Tost, L., Young, H., 2017. Moving Beyond the Virtual Museum: Engaging Visitors Emotionally. In: 23rd International Conference on Virtual Systems and Multimedia (VSMM 2017), ISBN 9781538644959.

Pescarin, S., 2014. Museums and Virtual Museums in Europe: Reaching expectations. SCIRES-IT, 4(1), pp. 131-140.

Pietroni, E., Pagano, A., Fanini, B., 2018. UX Designer and Software Developer at the Mirror: Assessing Sensory Immersion and Emotional Involvement in Virtual Museums. Studies in Digital Heritage,2(1), pp. 13-41, doi.org/10.14434/sdh.v2i1.24634.

Povroznik, N., 2018. Virtual Museums and Cultural Heritage: Challenges and Solutions. In: Mäkelä, E., Tolonen, M., Tuominen, J. (eds) DHN 2018, Digital Humanities in the Nordic Countries 3rd Conference, Helsinki, Finland, March 79, 2018, pp. 394-402.

Pratali Maffei, S., Canevese, E., De Gottardo, T., 2019. The real in the virtual. The 3D model in the Cultural Heritage sector: the tip of the iceberg. In: Int. Arch. Photogramm. Remote Sens. Spatial Inf. Sci., Vol. XLII-2/W9, pp. 615-621, doi.org/10.5194/isprs-archives-XLII-2-W9-615-2019.

Remondino, F., Georgopoulos, A., González-Aguilera, D., Agrafiotis, P., 2018. Latest Developments in RealityBased 3D Surveying and Modelling. MDPI, Basel, $\mathrm{CH}$, doi.org/10.3390/books978-3-03842-685-1-8.

Tschirschwitz, F., Büyüksalih, G., Kersten, T. P., Kan, T., Enc, G., Baskaraca, P., 2019. Virtualising an Ottoman fortress Laser scanning and 3D modelling for the development of an interactive, immersive Virtual Reality application. In: Int. Arch. Photogramm. Remote Sens. Spatial Inf. Sci., Vol. XLII-2/W9, pp. 723-729, doi.org/10.5194/isprs-archives-XLII-2-W9-7232019. 